\title{
Entre texte et contexte : la fiction historique et la fiction à substrat professionnel
}

Jean-Louis Trouillon

\section{(2) OpenEdition}

\section{Journals}

Édition électronique

URL : http://journals.openedition.org/asp/778

DOI : $10.4000 /$ asp.778

ISBN : 978-2-8218-0400-5

ISSN : 2108-6354

Éditeur

Groupe d'étude et de recherche en anglais de spécialité

Édition imprimée

Date de publication : 1 janvier 2005

Pagination : 51-65

ISSN : 1246-8185

\section{Référence électronique}

Jean-Louis Trouillon, « Entre texte et contexte : la fiction historique et la fiction à substrat professionnel », ASp [En ligne], 47-48 | 2005, mis en ligne le 16 février 2010, consulté le 19 avril 2019. URL : http://journals.openedition.org/asp/778 ; DOI : 10.4000/asp.778

Ce document a été généré automatiquement le 19 avril 2019

Tous droits réservés 


\title{
Entre texte et contexte : la fiction historique et la fiction à substrat professionnel
}

\author{
Jean-Louis Trouillon
}

\section{Introduction}

1 L'objectif de cette étude n'est pas de savoir si, pour la communauté des chercheurs LANSAD, la fiction historique $(\mathrm{FH})$ relève ou non, de la fiction à substrat professionnel (FASP), dont il a été montré que les contours étaient par nature imparfaitement définis (Isani 2004 : 34). Il s'agit plutôt de profiter des avancées proposées par la recherche portant sur la FASP pour étudier la FH à partir d'une approche LANSAD et de montrer qu'au-delà des différences de surface il existe une possibilité de convergence, et ce en particulier au niveau du regard didactique que l'on peut porter sur les deux genres.

2 Il semblerait en effet quelque peu vain de vouloir rattacher nolens volens la FH à la FASP. Si cette dernière n'a été que récemment reconnue en tant que genre (Petit 1999) le genre FH est, quant à lui, reconnu depuis au moins deux siècles : dans son essai sur Claude et Néron Diderot traitait déjà, de façon fort critique d'ailleurs, du roman historique : « [...] le roman historique est un mauvais genre: vous trompez l'ignorant, vous dégoûtez l'homme instruit, vous gâtez l'histoire par la fiction et la fiction par l'histoire » (Diderot 1778 cité par Boeglin 1999).

3 Il convient de rappeler dès à présent ce que l'on entend par FH. Les spécialistes s'accordent à la définir comme un genre hybride, à cheval entre ces deux domaines que sont la fiction et l'histoire. Morère parle même d'aporie: «la notion même de roman historique semble une aporie. Alors que l'histoire prétend tenir un discours vrai sur le passé, le roman crée un univers fictif. » (cité par Gaston-Lagorre 2002). Le Pellec, quant à lui, préfère le terme d'oxymore tout en justifiant ce choix terminologique : «Le Roman historique relève, par son appellation et sa nature de l'oxymoron [...] sans doute parce que, conjoignant les contraires, elle est emblématique de la nécessité actuelle de repenser 
les polarités et les incompatibilités que nous inculqua la tradition. » (Gaston-Lagorre 2002).

4 Notre point de vue LANSAD n'est pas de prendre part à ce débat et il nous suffira de considérer comme FH toute fiction où le récit inventé prend le pas sur le discours historique, l'histoire connue, c'est-à-dire l'histoire reconnue par l'institution universitaire, restant le référent obligé du récit. Histoire est à prendre au sens le plus large qui soit puisque la discipline accepte et adopte comme objet d'étude toute activité humaine dès lors qu'elle se situe dans le passé : le cadre pourra donc être politique, social, militaire ou religieux, se situer dans un milieu professionnel donné ou encore reposer sur des relations familiales ${ }^{1}$.

\section{Les différences}

5 La première différence tient sans aucun doute aux origines des deux genres, et dans le temps, et dans l'espace. Si l'on s'en tient à une définition orthodoxe, deux des caractéristiques majeures de la FASP (Petit 1999) sont d'une part d'être « un genre exclusivement anglophone » et d'autre part un genre qui s'est développé surtout dans les deux dernières décennies du $\mathrm{XX}^{\mathrm{e}}$ siècle.

6 En revanche, la FH sous toutes ses formes remonte dans nos civilisations occidentales, au moins à la fin du premier millénaire de notre ère, si l'on choisit d'éliminer les auteurs de l'Antiquité pour lesquels il nous est souvent difficile de faire la différence entre le mythe et la réalitée .

7 Par ailleurs, même si la critique considère généralement que le roman historique est né en tant que genre avec Waverley de Walter Scott en 1814, de nombreux pays ont très tôt proposé des exemples maintenant célèbres de ce genre littéraire.

8 En 1605 Cervantès faisait paraître Don Quichotte, en tant que roman parodique certes, mais qui reste tout de même par ailleurs une fresque sociale et historique d'une époque donnée. Il aurait, semble-t-il, travaillé à la rédaction d'un véritable roman historique jamais achevé ; il est également l'auteur d'une pièce historique, La Numancia, située à l'époque romaine.

9 Ceci nous rappelle, en littérature de langue française, bon nombre de pièces historiques de Corneille et Racine au XVII ${ }^{e}$ siècle. En France encore, Mme de Lafayette a publié $L a$ Princesse de Clèves en 1678, et c'était là son troisième ouvrage que l'on peut qualifier d'historique.

10 En Allemagne, on se préoccupe de définir le roman historique comme genre dès 1788 (Mühlberger \& Habitzel 2001). Tolstoï publie Guerre et paix en 1869. Le film Quo Vadis, tourné en 1951 par Mervin Leroy, est adapté d'un roman de Henryk Sienkiewicz publié en Pologne en 1931. Plus récemment, en Italie, Le Nom de la rose d'Umberto Eco s'inscrit également dans la tradition du roman historique.

11 L'on pourrait multiplier les exemples, mais ce serait inutile : rien qu'en Allemagne, en effet, on compte 6300 romans historiques écrits entre 1780 et 1944 (Mühlberger \& Habitzel 2001). Il s'agissait simplement de montrer que le genre FH dépassait, de loin et depuis longtemps, les limites de la production anglophone.

12 La deuxième différence tient aux différentes représentations, littéraires ou autres, de la FH, plus nombreuses semble-t-il, que pour la FASP. Si le roman, le cinéma, la télévision et 
la bande dessinée sont des supports dans les deux cas, on peut pratiquement arrêter là la typologie pour ce qui est de la FASP, alors que le théâtre, la poésie, l'opéra, voire la peinture, ont été, au cours des siècles passés, mis à contribution pour ce qui relève de la $\mathrm{FH}$.

En restant cette fois-ci dans le domaine anglo-saxon et en ne citant que les exemples les plus connus, si l'on ne peut sans doute retenir dans le cadre de notre propos la saga en vers de Beowulf qui tient de la légende plus que de l'histoire, la poésie héroïque The Battle of Maldon (fin du $\mathrm{X}^{\mathrm{e}}$ siècle) initie une série qui s'est poursuivie jusqu'à nos jours. On pourrait citer au XIXe siècle Tennyson et sa Charge of the Light Brigade. Plus récemment encore Roman Wall ou $O$ What is that Sound de W. H. Auden relèvent sans aucun doute de notre propos.

Au théâtre, il suffira de rappeler la série des pièces historiques de Shakespeare situées aussi bien dans l'Antiquité classique que dans le passé du Royaume.

A livets soient souvent rédigés en italien pour répondre aux canons de l'époque, on peut citer Dido and Aeneas de Purcell en 1689 ou, si l'on trouve que l'Énéide relève plus de la légende que de l'histoire, on relèvera quelques années plus tard, le Julius Caesar de Handel en 1723.

Pour ce qui est de la chanson à substrat historique on trouve beaucoup moins d'exemples, dans les pays anglophones tout au moins ${ }^{3}$. Un des rares exemples pourrait être Children's Crusade de Sting. Il existe néanmoins une forte tradition dans d'autres civilisations, en Europe centrale et orientale en particulier ${ }^{4}$.

17 A contrario, si l'on songe à la FASP, une fois sortis du roman, du film, de la série télévisée et de la bande dessinée, ce qui est déjà appréciable comme diversité, il n'y a guère qu'au théâtre sans doute que l'on peut trouver quelques exemples, et ce dans le seul domaine s'approchant du juridique: The Merchant of Venice de Shakespeare ou plus récemment Twelve Angry Men de Reginald Rose qui a connu différents avatars puisqu'il s'agit d'abord d'une television play avant d'être le film de Sydney Lumet, puis de devenir une pièce de théâtre en 1964. On ne trouve ni technothriller chanté ni fiction médicale à l'opéra. Même si l'on peut proposer des exemples d'opéra à connotations politico-militaires, il ne s'agit pas réellement de FASP mais plutôt de sujets servant de simples toiles de fond.

Enfin, pour ce qui est de la bande dessinée, les séries américaines Beetle Bailey et Sad Sack's Army Life sont les deux seuls exemples d'ampleur d'une FASP militaire. Si l'auteur de Beetle Bailey, Mort Walker, dont les ouvrages sont en vente dans tout PX, est un personnage fort apprécié par les militaires américains, la série Sad Sack's Army Life est publiée dans le format comics et s'adresse théoriquement à un public d'enfants et adolescents. C'est là un des rares cas où la FASP vise un public de non-adultes ${ }^{5}$. En revanche, une partie importante de la FH s'adresse explicitement à un lectorat d'enfants et adolescents, à partir de l'âge de neuf ans :

les auteurs de la littérature pour la jeunesse sont principalement

- des enseignants,

et parmi eux, plutôt des professeurs de français (en majorité) et d'histoire-

géographie

- des femmes. (Arcoutel-Thoreau 2004)

19 Une troisième différence tient aux auteurs. Si, dans les deux cas, on trouve des spécialistes de la discipline (médecins, juristes, universitaires d'une part et historiens d'autre part comme par exemple Pierre Grimal avec Les Mémoires d'Agrippine), la plupart de ces «professionnels auteurs» (Petit 1999: 63) de FASP sont des spécialistes de ce 
genre, pratiquement à l'exclusion de tout autre. Il est néanmoins possible de trouver des contre-exemples comme celui de Franck G. Slaughter, bien connu pour ses romans de FASP médicale, qui a aussi écrit au moins un roman historique, The Purple Quest. À l'inverse, les auteurs de FH peuvent être également par ailleurs auteurs de fiction traditionnelle: Daphné du Maurier nous propose au milieu de ses nombreux romans The Glass Blowers. Ken Follett, bien connu pour ses romans policiers et d'espionnage, est également auteur de The Pillars of the Earth, fresque des XII ${ }^{e}$ et XIII ${ }^{e}$ siècles : «My publishers were a little nervous about such a very unlikely subject but paradoxically, it is my most popular book. It's also the book I'm most proud of. » (Follett 2005)

Une quatrième différence tient à l'approche du domaine et de l'intrigue : la FH se prête parfois à un traitement parodique, humoristique, comme par exemple, dans le cas du roman, la série Flashman écrite à partir de 1969 par George MacDonald Fraser. Cette série met en scène le big bully inventé par Thomas Hugues dans son Tom Brown's Schooldays, une fois que Tom Brown a été renvoyé de Rugby, nous faisant ainsi découvrir de façon comique, et parfois un peu leste, l'épopée coloniale britannique au XIXe siècle. La FASP humoristique, tout au moins en littérature ${ }^{6}$, ne concerne guère que le monde universitaire avec divers ouvrages de David Lodge et de Tom Sharpe.

En image, on peut mentionner la saga télévisée britannique The Black-Adder avec l'acteur comique Rowan Atkinson dans le rôle du personnage principal. Un des épisodes de la série se moque, entre autres, d'habitudes langagières archaïques, ce qui montre bien que les pratiques véritables de l'époque n'avaient sans doute que bien peu à voir avec le discours moderne généralement adopté :

Black-Adder: Is this Putney?

Old Woman: Indeed, that it be.

Black-Adder: Yes it is, not that it be ! You don't have to speak in that stupid voice to me, I'm not a tourist. (Curtis \& Elton 1992)

Cette brève citation nous rappelle que dans le cadre de la FH nous sommes souvent fort éloignés du trait discursif proposé comme donnée fondamentale de la FASP : « une langue reproduisant les pratiques langagières (lexicales et discursives) de ce milieu »(Petit 2004 : 5). Contrairement à la FASP qui reste une des sources du discours réaliste, la FH ne retranscrit pas le discours parlé à l'époque considérée, ce qui est d'ailleurs logique, les récits se situant parfois dans un pays dont bien peu de lecteurs seraient capables d'appréhender la langue. Nous verrons d'ailleurs infra, en faisant appel à Shakespeare, que la recherche de l'archaïsme de la langue n'est pas un garant d'exactitude vis-à-vis de l'époque étudiée. On pourrait en revanche considérer que le discours modernisé proposé par l'auteur de FH est proche du discours de l'historien contemporain sur la période en question.

Cette vision purement parodique, et sans aucun souci de vérité historique ou didactique, semble absente de la FASP, même si certaines œuvres s'en rapprochent comme le MASH (1968) de Richard Hooker adapté au cinéma par Robert Altman en 1970, mais ni le roman ni le film ne peuvent prétendre à l'appellation FASP. Il ne suffit pas en effet que l'action et l'intrigue se déroulent dans un milieu professionnel, et pour MASH le milieu est double, à la fois médical et militaire, pour que l'œuvre puisse entrer dans le cadre de la FASP puisque « l'intrigue est, dans la FASP, de nature endogène : elle se forme à l'intérieur du réel professionnel qui l'engendre » (Petit 1999 : 68), ce qui n'est pas vraiment le cas ici. Coluche ont, à quelques années d'intervalle, incarné le personnage du Roi Dagobert. Il est 
possible également de rappeler la longue tradition du comique troupier, initialement sur scène de Music-Hall puis au cinéma.

La différence majeure enfin, celle qui permet sans doute de déclarer sans contestation possible qu'il s'agit bien de deux genres différents, c'est le rapport de l'auteur avec le milieu de l'intrigue. Dans la FASP c'est cette relation serrée qui est à l'origine de l'intérêt que nous lui portons puisqu'elle nous permet de découvrir le dit milieu et d'en donner un aperçu à nos étudiants (Petit 2004 : 5).

A contrario, la FH est généralement due à la plume d'un romancier, qui certes se sera fortement documenté sur l'époque ainsi qu'on peut le découvrir grâce à la lecture des programmes de certains writing courses (Reichard 1999), mais qui donnera une vision nécessairement recomposée, romancée, des relations entre les individus. Même lorsque la fiction historique est l'œuvre d'un historien spécialiste de la période et du milieu considérés, on ne peut admettre qu'il y ait connaissance directe et contemporaine de ce milieu : le péritexte cite comme preuve de compétence le cas de Gary Jennings qui a vécu douze ans au Mexique avant d'écrire le premier tome de sa trilogie Aztec, mais le critique universitaire ne peut s'empêcher de remarquer que quatre cents ans séparent le moment du récit de celui de l'écriture! Si la géographie physique et la météorologie n'ont guère changé au cours de cet intervalle, il n'en va évidemment pas de même de la civilisation aztèque et l'on s'interroge sur le degré de naïveté des auteurs de ce péritexte ou du niveau d'ignorance qu'ils supposent chez l'acheteur.

\section{Les ressemblances}

En dépit de ces différences apparemment flagrantes entre les deux genres, on peut néanmoins noter aussi un certain nombre de ressemblances de la $\mathrm{FH}$ avec les caractères principaux de la FASP, permettant ainsi une approche commune.

La première ressemblance relève de questions que la critique pose quant à la classification et la définition de nos deux genres.

Notre communauté LANSAD s'est interrogée pour savoir ce qui relevait de la FASP et ce qui n'en était pas: est-ce que l'absence de l'un des critères rend nulle et non avenue l'appartenance au genre ? Faut-il proposer une définition plus large qui, par exemple, ne tiendrait compte ni du temps ni de l'espace? Certains ouvrages de Balzac ${ }^{7}$ ou de Zola ${ }^{8}$, pour ne citer que les écrivains les plus connus, pourraient alors à juste titre prétendre à l'étiquette FASP.

Nos collègues spécialistes de la FH se sont également interrogés sur l'appartenance au genre et l'on trouve par exemple les tenants d'une ligne que l'on pourrait qualifier de dure selon laquelle il serait impératif que l'auteur n'ait pas personnellement connu l'époque dont il traite: pour Walter Scott déjà, il fallait au moins un écart de deux générations entre le fait et sa narration (Robinson 2004). Une autre école décrètera qu'un écart de $\mathrm{n}$ années entre l'écriture de l'ouvrage et la période décrite suffit à permettre le rattachement au genre, ce qui permet d'accepter comme roman historique des ouvrages tels que The Naked and the Dead de Norman Mailer, ou en France Le Feu de Barbusse ou Les Croix de bois de Dorgelès.

31 On peut aussi faire remarquer qu'une bonne partie de la critique, en s'inscrivant dans la lignée que l'on a nommée humaniste du roman historique, porte un regard de type marxiste sur la fiction historique : 
Avec le roman historique, ce n'est pas à la simple naissance d'un genre littéraire que l'on assiste, mais à la dissolution par la critique historique matérialiste d'un univers d'humanisme bourgeois voué à croître et à dépérir dans les limites temporelles d'un même siècle, laboratoire saturé d'expériences politiques. (Floccari 2000)

la lutte du bien contre le mal, du petit contre le puissant, de l'individu contre l'institution,
de la démocratie contre la dictature. Des milliers de romans d'espionnage écrits lors de la
guerre froide, précédant de peu notre période de référence donc, peuvent ainsi être lus dans ce sens. Néanmoins, l'aspect «action », et la force cathartique que cette dernière entraîne, prédominent certainement dans les motivations du lecteur, en prenant ainsi le pas sur la réflexion géopolitique, voire en l'effaçant complètement aux yeux des lecteurs dénués de conscience politique. diffusion et d'adaptation (du roman à l'écran en particulier). Dans les deux cas nous nous trouvons devant une littérature que l'on peut qualifier de volumineuse, aussi bien parce que les ouvrages sont souvent eux-mêmes fort longs, que parce qu'ils sont imprimés et diffusés à grande échelle, avec des politiques éditoriales très proches pour tout ce qui relève du péritexte. Les remarques proposées par Petit peuvent être reproduites in extenso pour le roman historique (il s'agit bien ici de roman et non de fiction en général, le roman étant le genre le plus fréquent). Les mots suivants qui caractérisent ThePillars of the Earth pourraient aussi bien s'appliquer à un ouvrage relevant de la FASP : « An extraordinary epic buttressed by suspense ... a mystifying puzzle ... a monumental masterpiece ... a towering triumph from a major talent! ( Follett 2005) et aussi « A seesaw of tension ... action, intrigue, violence and passion ... A novel that entertains, instructs and satisfies on a grand scale! » (ibid.).

Les données chiffrées prouvent le succès du genre : ce même ouvrage Pillars of the Earth a été numéro un des ventes au Royaume-Uni et en Italie, et, lors d'un sondage effectué par la chaîne de télévision ZDF, a été élu troisième livre le plus populaire d'Allemagne le $1^{\mathrm{er}}$ octobre 2003 par 250000 lecteurs (en troisième position après The Lord of the Rings et la Bible). Il se vend toujours annuellement à 100000 exemplaires aux États-Unis (Follett 2005).

Un classement établi par France Loisirs place le roman historique en $3^{\mathrm{e}}$ position de ses ventes (environ $25 \%$ ) après le roman général et le roman passion, avant les récits régionaux, les auteurs classiques et les romans drôles ${ }^{9}$. La terminologie proposée ici est celle de France Loisirs et cette donnée est sans doute à manipuler avec précaution: 
France Loisirs classe par exemple Autant en emporte le vent dans les romans passion et non les romans historiques.

La troisième ressemblance, qui découle immédiatement de la donnée précédente, tient à la vigueur du genre. Que ce soit à l'écrit ou en image, les productions abondent. En 1997, $36 \%$ de tous les ouvrages de fiction passés en revue par le New York Times relevaient de la fiction historique (Robinson 2004). On peut s'interroger pour savoir si les raisons du succès de ces deux genres ont une origine commune. Il faudrait pour cela procéder à une analyse fine du lectorat et de ses motivations. De simples sondages opérés auprès de lecteurs de romans historiques semblent confirmer ce que l'on pouvait supposer quant à l'attrait du genre. Il s'agirait d'une vision, que l'on pourrait baptiser populaire, de l'histoire ; celle-ci n'apparaît plus comme une discipline universitaire désincarnée mais devient le produit, non seulement de décisions humaines, mais aussi de passions comme l'amour ou la haine. Le lecteur éprouverait par ailleurs un intérêt quasi pathologique pour un temps révolu, vu comme préférable par rapport aux incertitudes de l'époque actuelle. L'on est très proche ici de ce qu'écrivait l'historienne et romancière Zoé Oldenbourg pour qui «le roman d'histoire correspond à un besoin réel de l'homme occidental spirituellement déraciné, privé de ses mythes, et avide de s'intégrer dans un passé humain » (citée par Boeglin 1999).

41 Ces idées sont confortées par les conseils en creative writing donnés aux auteurs tentés par l'écriture :

Your reader is not looking for a history textbook. She knows where those are shelved in the bookstore and if she wanted one, she would buy one. She doesn't want a lengthy treatise about jousting, feasting, armor, herbs, the Crusades, or the king's problems with his vassals. She's buying your novel because she wants to read a compelling story about fascinating people. (Thacker Meinhardt 2004)

L'image, par le biais de la bande dessinée, des films ou des séries télévisées historiques, ajoute en outre une facilité que n'offre pas la lecture, ne serait-ce que par une représentation visuelle en trois dimensions, en couleur et parfois en mouvement lorsqu'il s'agira de films ou séries télévisées, de l'ensemble des cadres de vie de l'époque considérée : vêtements, habitat, ustensiles, armes, moyens de transport, etc.

\section{La fiction historique et la didactique en secteur LANSAD}

43 C'est bien là en effet le point majeur qui est à l'origine de la question des rapports entre les deux genres: quel est le regard que porte le didacticien sur la $\mathrm{FH}$ et plus particulièrement sur le roman? De même que la FASP juridique ou économique, pour ne citer que les principales représentantes du genre, méritent de figurer dans nos cours LANSAD grâce à la qualité de leurs références professionnelles, cette question préliminaire doit être posée afin de permettre éventuellement à l'enseignant LANSAD de s'approprier la FH et de l'utiliser sans crainte de faire fausse route.

En dépit de ce que pensait Diderot (cf. supra), la valeur didactique du roman historique a été très tôt perçue. Macaulay déjà, en 1828 , défendait l'idée que le roman historique était préférable au roman car ce qui était narré s'était réellement produit et présentait donc un intérêt complémentaire aux yeux du lecteur (Oergel 2001). En France, Flaubert reconnaissait que «seuls les romans historiques peuvent être tolérés parce qu'ils 
enseignent l'histoire » (cité par Boeglin 1999). Quant à Dumas, lui-même auteur de FH pourtant largement fictive, il déclarait :

Notre prétention en faisant du roman historique est non seulement d'amuser une classe de nos lecteurs, qui sait, mais encore d'en instruire une autre qui ne sait pas, et c'est pour celle-là particulièrement que nous écrivons. (cité par Boeglin 1999)

Cette valeur didactique est toujours mise en avant à l'heure actuelle comme argument de vente : « a novel that instructs » a déjà été cité à propos de The Pillars of the Earth.

Dans ce cadre didactique, même si on trouve parfois des phrases du genre : «for decades, knowledgeable teachers of history have used 'historical fiction' to heighten student awareness of the people and times under study $»^{10}$, le genre n'intéresse guère nos collègues universitaires français. Peut-être éprouvent-ils une certaine crainte par rapport aux réactions éventuelles de l'institution, même si certains grands noms n'hésitent pas aborder le sujet comme, par exemple, Le Roy Ladurie : «Je suis très tenté par le roman historique : je suis à la frontière des deux, mais j'ai un peu peur des douaniers » (Boeglin 1999).

En revanche, plusieurs de nos collègues enseignants du second degré, historiens et professeurs de lettres, utilisent, souvent en commun, le roman historique et en particulier le roman historique pour la jeunesse (Comelli 2002). Les raisons de leur choix sont de trois ordres.

8 En premier lieu, il s'agit de raisons purement linguistiques comme par exemple l'étude du temps de l'écriture. En deuxième lieu, est mentionnée la formation aux capacités analytiques et critiques de l'apprenant : la notion de point de vue et donc d'objectivité et de subjectivité, les notions de vrai et de vraisemblance, la question de la réalité et sa représentation, tous ces phénomènes peuvent évidemment être abordés à partir de la FH. En troisième lieu enfin, on trouve évidemment la connaissance première de la discipline Histoire, non seulement par la richesse documentaire du roman historique, voire de la bande dessinée historique (il a été rappelé supra que nombre d'historiens sont auteurs de FH) mais aussi par l'apprentissage de la distinction entre texte et récit historique.

L'intérêt qu'éprouve l'élève adolescent pour cette approche de l'histoire se fera souvent par le biais de l'identification au héros. Toutefois, dans le cadre de notre situation d'enseignant dans le secteur LANSAD ce dernier point n'existe pratiquement plus si nous travaillons avec des romans de type grand public et non destinés à la jeunesse.

Que nous reste-t-il alors? Il faudrait en premier lieu rappeler les objectifs de notre enseignement dans le cadre des sciences humaines : les étudiants sont inscrits en Licence, éventuellement en Master, d'Histoire.

1 Pour les besoins de notre étude nous découperons la discipline Histoire selon trois axes : chronologique (ce que les spécialistes appellent histoire ancienne, histoire du Moyen-Âge, histoire moderne et histoire contemporaine), géographique (histoire d'un pays, d'une région ou d'une ville) et thématique (cf. supra note 1). L'historien travaillera sur un sujet recoupant ces trois axes, par exemple l'histoire de la condition féminine (thème) dans l'Angleterre (géographie) victorienne (époque).

52 La multitude des thèmes considérés par les historiens fait que l'enseignant LANSAD ne saurait ni rechercher une exhaustivité thématique, ni trouver facilement un substrat lexical commun aux différentes spécialités, substrat qui permettrait de proposer au moins une terminologie spécifique utilisable ultérieurement quelle que soit la spécialité choisie.

Le travail préparatoire à l'établissement d'un cursus effectué à partir des matériaux d'étude de l'historien permet de constater que ces matériaux relèvent de deux ordres 
bien distincts posant un double problème linguistique: la première catégorie est constituée par les sources primaires (ou documents d'époque, registres, traités ou journaux par exemple) et la deuxième catégorie est celle des documents rédigés a posteriori par les historiens spécialistes de l'époque. pour ne citer que les domaines les plus fréquents, les motivations des étudiants en histoire sont rarement de type instrumental : bien rares sont les étudiants, tout au moins dans le cadre de la Licence, et bien souvent encore en Master, qui ont besoin de consulter des documents écrits en anglais. Le temps réservé dans les années de Licence à l'étude de l'histoire des pays de langue anglaise est relativement restreint et n'incite donc guère à de longues recherches. Même lorsque ces programmes sont consacrés aux pays anglophones, les bibliographies proposées aux étudiants citent plus souvent des historiens français (en particulier C. Fohlen ou A. Kaspi pour les États-Unis et R. Marx ou F. Bédarida pour le Royaume-Uni) que des historiens anglophones et les plus importants de ces derniers, tout au moins aux yeux de l'historiographie française, ont de toute façon été traduits en français comme D. Boorstin et son Histoire des Américains ${ }^{11}$. Les historiens anglophones spécialistes de l'histoire de France cités dans les bibliographies le sont généralement en traduction française ${ }^{12}$. L'objectif LANSAD ne se contente heureusement pas documents professionnels, l'apprentissage de la vie dans un milieu anglophone fait également partie de notre mission. Or, bien rares sont les étudiants en histoire qui souhaitent participer à des échanges de type ERASMUS ${ }^{13}$ et bien rares aussi ceux qui choisiront à partir de leurs années de Master de se spécialiser dans l'étude des pays anglophones.

L'enseignant LANSAD peut donc à juste titre être amené à renoncer à un but immédiatement utilitaire de la langue en tant que telle. Il lui appartient de se fixer de nouveaux objectifs comme par exemple l'éveil à une civilisation étrangère d'une part et le développement de l'esprit critique d'autre part. On retrouve là une dimension traditionnelle de l'enseignement des langues, en particulier dans le second degré.

Cette dimension culturelle, se révèle facile à appréhender grâce à la FH puisqu'elle est l'un des objectifs que se donnent les auteurs qui n'hésitent pas à s'enorgueillir de leur capacité à montrer un monde différent du nôtre ; c'est ainsi que Follett, écrit sans trop de modestie à propos de The Pillars of the Earth :

It recreates, quite vividly, the entire life of the village and the people who live there. You feel you know the place and the people as intimately as if you yourself were living there in the middle ages. (Follett 2005) 
au-delà d'un simple apprentissage de la langue, d'une langue qui reste d'ailleurs à définir comme il a été montré supra, une approche faisant aussi appel à la FH permet en particulier d'expliquer certains stéréotypes et de prendre du recul par rapport à une vision trop nationaliste de tel ou tel événement.

61 La bataille d'Azincourt et les préparatifs qui la précèdent, tels qu'ils sont imaginés par Shakespeare dans Henry $V$, et illustrés par le cinéma nous serviront d'exemple.

Grâce à la $\mathrm{FH}$, la représentation ou la visualisation du décor permettent un commentaire, des exemples d'analyse pouvant être proposés à partir des remarques de spécialistes de l'époque sur le film lui-même :

When Laurence Olivier's [...] Henry $V$ was first disclosed to a group of Oxford's impassive Shakespeare pundits [...] a woman specialist insisted that all the war horses which take place in the Battle of Agincourt should have been stallions. (Agee 1946)

63 La difficulté réelle de la langue, dont il faut noter que, bien qu'archaïque, elle est d'ailleurs elle-même anachronique par rapport à l'époque décrite, est atténuée par une étude en parallèle des films tournés à partir de la pièce. Le travail approfondi d'analyse sur les notions de point de vue, de nationalisme, d'objectivité, de description du réel, auquel la comparaison entre le texte écrit et les scènes filmées donne lieu dépasse rapidement le texte et permet ainsi d'éviter la critique facile qui risquerait d'être adressée à l'enseignant accusé de se contenter d'enseigner, et c'est une véritable occasion de le dire, «la langue de Shakespeare » : si la langue reste l'objectif désigné, elle devient aussi un moyen, c'est là ce que nous permet la FH.

\section{Conclusion}

Puisque le questionnement proposé porte sur les relations entre FASP et FH, il semble logique de répondre que la FH est à l'historien ce que la FASP est au spécialiste de la profession considérée c'est-à-dire une fiction qui se greffe sur une représentation du réel.

Si l'on décide d'ignorer le problème philosophique et linguistique que pose cette description de la réalité observable par le langage, ce qui rapproche étroitement les deux genres, c'est d'abord le choix d'un genre par l'auteur, ensuite la similitude probable de motivation de la part du lecteur, et enfin le regard de la critique universitaire et en particulier du spécialiste LANSAD.

Le parallèle principal à établir repose bien sur l'introduction de l'œuvre de fiction dans un cours LANSAD. Quant à la pratique qui en résulte, les apports dont bénéficie l'apprenant se situent à des niveaux différents: meilleure connaissance du milieu professionnel et de son langage dans le cas de la FASP, alors que la FH ne tient nullement compte du milieu professionnel de l'historien ${ }^{14}$ mais se porte sur les domaines d'étude de ce dernier, en permettant ainsi une approche complémentaire, souvent plus vivante que le document scientifique, et donc généralement plus motivante pour l'apprenant. 


\section{BIBLIOGRAPHIE}

\section{Corpus de référence}

Auden, Wystan H. 1968. Selected Poems. Londres : Faber Paperbacks.

Balzac, Honoré de. 1972 [1829]. Les Chouans ou la Bretagne en 1789. Paris : Gallimard/Folio.

Balzac, Honoré de. 1961 [1843]. Illusions Perdues. Paris : Classiques Garnier.

Baker, George. Sad Sack' Army Life. 10 avril 2005 <http://www.sadsack.net>.

Barbusse, Henry. 1988 [1916]. Le Feu. Paris : Le Livre de Poche LGF.

Branagh, Kenneth. 1989. Henry V. MGM/UA.

Campbell-Risen, Meredith. 2000. Righteous Warriors. Independence, MO : Two Trails Publishing.

Cervantes Saavedra, Miguel de. 1581. La Numancia. <http://www.cervantesvirtual.com/servlet/ SirveObras/cerv/01305008611682944755802/index.htm>.

Cervantes Saavedra, Miguel de. 1967 [1605-1615]. El Ingenioso Hidalgo Don Quijote de la Mancha. Madrid : Espasa-Calpe, S.A.

Charlier, Jean-Michel. Depuis 1947. Les Aventures de Buck Danny. Bruxelles : Dupuis.

Chase-Riboud, Barbara. 1994 [1979]. Sally Hemmings: A Novel. New York : Ballantine Books.

Chevalier, Pierre. 1963. Le Bon Roi Dagobert. Cineurop productions, Filmeric.

Curtis, Richard \& Ben Elton. 1992. «Bells » Black-Adder II, BBC Enterprises Ltd.

Dorgelès, Roland. 1919. Les Croix de bois. Paris : Albin Michel.

Du Maurier, Daphné. 1963. The Glass Blowers. Londres : Gollancz.

Eco, Umberto. 1983. Le Nom de la rose. Paris : Le Livre de Poche LGF.

Follett, Ken. 1989. The Pillars of the Earth. New York : William Morrow.

Grimal, Pierre. 1992. Les Mémoires d'Agrippine. Paris : Éditions de Fallois.

Handel, George F. 1723. Julius Caesar. Nuova Era.

Hooker, Richard. 1968. MASH. New York : William Morrow.

Jennings, Gary. 1980. Aztec. New York : Buccaneer Books.

Levinson, Barry. 1997. Wag the Dog. Warner Bros.

MacDonald Fraser, George. 1970 [1969]. Flashman. Londres : Pan Books Ltd.

Mailer, Norman, 1976 [1949]. The Naked and the Dead. Frogmore : Panther Books Ltd.

Olivier, Laurence. 1944. Henry V. A Two Cities Film.

Purcell, Henry. 1689. Dido and Aeneas. Virgin Veritas.

Risi, Dino. 1984. Le Bon Roi Dagobert. Gaumont.

Rose, Reginald.1955. Twelve Angry Men. CBS Studio One. 
Scott, Walter. 1985 [1814]. Waverley. Londres : Penguin Classics.

Shakespeare, William. 1966 [1598]. The Merchant of Venice. Oxford : Oxford University Press.

Shakespeare, William. 1966 [1599]. Henry V. Oxford : Oxford University Press.

Sienkieviewicz, Henrik. 1931. 10 avril 2005 Quo Vadis. <http://www.gutenberg.org/etext/2853>.

Slaughter, Franck G. 1965. The Purple Quest A Novel of Seafaring Adventure in the Ancient World. New York : Doubleday.

Sting. 1985. « Children's crusade ». Dream of the Blue Turtles. Uni/A\&M.

Tennyson, Alfred Lord. 1994. The Works of Alfred Lord Tennyson. Ware : Wordsworth Editions Ltd.

Tolstoï, Léon. 1972 [1869]. Guerre et paix. Paris : Le Livre de Poche LGF.

Yourcenar, Marguerite. 1980 [1951)]. Les Mémoires d'Hadrien. Paris : Gallimard, collection Folio.

Walker, Mort. Beetle Bailey. 10 avril 2005. <http://www.kingfeatures.com/features/comics/ bbailey/about.htm>.

\section{Références}

Agee, James. 2005. « Review of Henry V for Time Magazine ». Olivier. 20 juillet $2005<$ http:// www.murphsplace.com/olivier/henry5. html>.

Arcoutel-Thoreau, Fabienne. 2004. «L'apprentissage de l'histoire à travers les romans historiques jeunesse ». 15 octobre 2004. Blois. 10 avril 2005 <http://www.ac-orleans-tours.fr/rdv-histoire/ ARCHIVES/2004/atelier-romans.htm>.

Boeglin, Edouard. 1999. « Le roman historique : littérature mineure ?». 13 juin 1999. Journal l'Alsace / Le Pays, 68945 Mulhouse. 10 avril 2005 <http://www.alsapresse.com/jdj/99/06/13/IRF/ article_12.html>.

Comelli, Dominique. 2002. « Rencontre 'Utiliser le roman historique en classe' ». Blois. 20 juillet $2005<$ http://ww3.ac-creteil.fr/hgc/spip/article-.php3?id_article=277>.

Floccari, Stéphane. 2000. « Georg Lukacs, les noces du roman et de l'histoire ». L'Humanité. 27 septembre 2000.

Follett, Ken. 2005. The Master Storyteller and Best-Selling Author. 10 juin 2005. Site officiel de l'auteur. 20 juillet 2005 <http://www.ken-follett.com/bibliography/ pillars.html>.

Gaston-Lagorre, Brigitte. 2002. « Le roman historique ». 25 octobre 2002. CDDP du Gers. 10 avril 2005 <http://www.crdp-toulouse.fr/cddp-32/html/formation/enligne/ Roman_historique.htm>.

Isani, Shaeda. 2004. «The FASP and the Genres within the Genre ». In Petit, Michel \& Shaeda Isani. Aspects de la fiction à substrat professionnel. Bordeaux : Université Victor Segalen Bordeaux 2, collection Travaux 2025, 25-36.

Lukacs, Georg. 1957 [1965]. Le Roman historique. Paris : Petite Bibliothèque Payot, 1965. Traduit de l'allemand.

Mühlberger Günter \& Kurt Habitzel. 2001. «The German historical novel (1780-1945): Aspects of a developing genre ». Amsterdam. 20 juillet $2005<$ http://histrom.literature.at/docs/kent.html>.

Oergel, Maike. 2001. « Wie es wirklish wurde »: The modern need for historical fiction, or the inevitability of the historical novel ». 1 juillet 2001 Amsterdam. 20 juillet $2005<$ http:// docserver.ingentaconnect.com/deliver/cw/rodopi/03046257/v51n1/s28/p435.pdf?

$\mathrm{fmt}=$ dirpdf\&tt=486\&cl=1088\&ini=connect\&bini=\&wis=connect\&ac=1\&acs=\&expires=1121948261\&cheksum=93B09A60F8DC431D33DA26F 
Petit, Michel. 1999. « La fiction à substrat professionnel : une autre voie d'accès à l'anglais de spécialité ». ASp, la revue du GERAS 23-26: 57-81.

Petit, Michel. 2004. «Quelques réflexions sur la fiction à substrat professionnel : du général au particulier ». In Petit, Michel \& Shaeda Isani. Aspects de la fiction à substrat professionnel. Bordeaux : Université Victor Segalen Bordeaux 2, collection Travaux 2025, 3-23.

Reichard, Sue. 1999. « Writing historical fiction for children ». 5 janvier 1999. Suite University. 20 juillet 2005. <http://www.suite101.com/article.cfm/childrens_writing/14324>.

Robinson, William. 2004. « A few thoughts on historical fiction ». 20 juillet $2005<$ http:// web.utk.edu/ wrobinso/590_lec_hisfic.html>.

Shentalinskaia, Tat'iana. 2002. « Major Pavlutskii: From history to folklore. From the materials collected by the American North-Pacific expedition ». SEEFA Journal - The Journal of the Slavic and East European Folklore Association Volume VII/1 : 3-21.

Thacker Meinhardt, Shelly. 2004 « Researching the historical Nnovel ». 20 juillet $2005<\mathrm{http}: / /$ www.shellythacker.com/researching.htm>.

Uglow, Nathan. 2002. « British historical novel ». 21 mars 2002. The Literary Encyclopedia. 10 avril 2005 <http://www.litencyc.com/php/stopics.php?rec =true\&UID=1213>.

\section{NOTES}

1. Sans prétendre à l'exhaustivité rappelons que le substantif « histoire » peut être déterminé de façon fort variable : on parle aussi bien d'histoire de la mort que d'histoire de la sexualité, du droit, de l'alimentation, de l'art, des sciences et techniques, du climat, du commerce, de l'économie, de l'éducation, de l'enfant, de la femme, de la famille, de l'esclavage, de la mode, du sport, du travail, des religions, des idées, et bien d'autres thèmes encore.

2. Si cette suggestion reste vraie pour des œuvres comme Lysistrata d'Aristophane, la question est néanmoins à débattre pour d'autres, en particulier Les Perses d'Eschyle.

3. Le film Wag the Dog fait bien appel à une chanson historique, mais il s'agit d'un faux.

4. Par exemple le chant rappelant l'épopée du Commandant Pavutskii en Sibérie.

5. Plusieurs bandes dessinées, surtout françaises et belges, à destination d'un public jeune, prennent pour cadre un milieu professionnel donné comme le Buck Danny de J. M. Charlier, pilote de chasse de l'Armée de l'Air américaine.

6. Des passages de la série Ally McBeal prêtent certes à rire ou sourire, mais on ne peut dire que dans son ensemble la série possède une vis comica particulière.

7. Par exemple, mais sans exclusive, Illusions Perdues pour le journalisme et pourquoi pas aussi l'industrie de la fabrication de la pâte à papier.

8. Plusieurs des ouvrages de la série des Rougon-Macquart.

9. France Loisirs, <http://www.franceloisirs.com/FL/index.htm>. Il s'agit ici d'un classement publié par France Loisirs à des fins publicitaires.

10. Cité en quatrième de couverture de Meredith Campbell, Righteous Warriors.

11. Le rayon Histoire de la bibliothèque de mon université ne propose qu'une seule revue rédigée en langue anglaise : Journal of the History of Ideas. Cinq titres sont proposés en espagnol. Environ $75 \%$ des étudiants choisissent l'anglais dans le cadre de leurs cours de langue.

12. L'Université Paris 1 propose par exemple: A. Cobban, Le sens de la Révolution française, traduction française, Julliard, 1984. Page Révolution Française, bibliographie. 28 octobre 2005 <http://ihrf.univ-paris1.fr/activ/JBRBiblioagreg.htm>. 
13. J'enseigne depuis trois ans en DEUG et Licence d'Histoire ; aucun de mes étudiants n'a ni émis le désir de participer à un échange ERASMUS ni souhaité accomplir une année d'étude aux ÉtatsUnis alors que ces deux possibilités leur sont offertes.

14. Ce milieu professionnel est en fait le milieu de l'enseignement et de la recherche, très largement commun à d'autres disciplines.

\section{RÉSUMÉS}

Après avoir rappelé les différences fondamentales entre les genres «fiction à substrat professionnel » et fiction historique, l'étude se propose de montrer les relations étroites qui les sous-tendent lorsqu'ils sont abordés sous l'angle LANSAD, et en particulier quant aux objectifs et modes éditoriaux, thèmes et lectorat. La dernière partie porte sur la convergence des deux genres dès lors qu'ils sont envisagés avec une approche didactique, et suggère une place à réserver à la fiction historique dans l'enseignement LANSAD.

After listing the major differences between Professionally-based fiction and Historical fiction as genres, the paper aims to show their underlying similarities when considered from an ESP point of view: purposes, publishing modes, subject matters and readership. The paper concludes with a presentation of the didactic convergence between the two genres, and suggests a way of using historical fiction in ESP courses.

\section{INDEX}

Mots-clés : anglais de spécialité, didactique, fiction à substrat professionnel, fiction historique, histoire

Keywords : didactics, ESP, historical fiction, history, professionally-based fiction

\section{AUTEUR}

\section{JEAN-LOUIS TROUILLON}

Jean-Louis Trouillon est maître de conférences habilité à diriger des recherches à l'Université de Perpignan Via Domitia. Son domaine de recherche porte sur divers aspect de l'anglais de spécialité et des langues pour spécialistes d'autres disciplines. Ses champs d'application recouvrent actuellement les sciences humaines et les forces armées. Il est auteur ou co-auteur de plusieurs didacticiels ; le dernier, paru en 2003 pour le ministère de la Défense, porte sur l'anglais opérationnel. jean-louis.trouillon@wanadoo.fr 